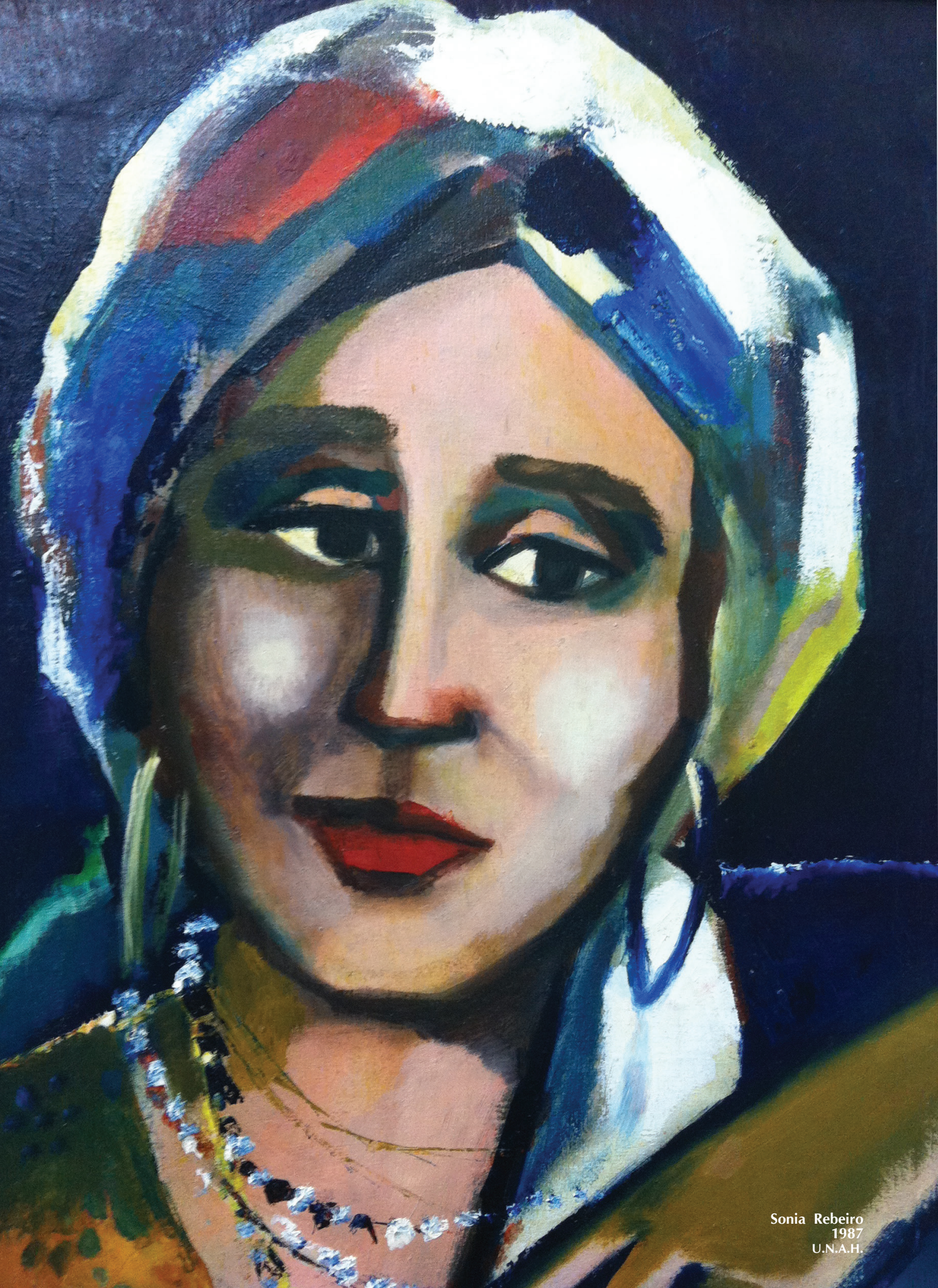




\section{EQUIDAD DE GENERO EN EL SISTEMA EDUCATIVO NACIONAL}

\section{Por: Abog . Cesar Agusto Ramos .}

Profesor Investigador, Instituto de Investigación Jurídica. Universidad Nacional Autónoma de Honduras.E-Mail: cesar_ramos63@yahoo.com

\section{Resumen}

La lectura del presente trabajo, nos llevará a identificar algunas formas exisistentes de desigualdad entre hombres y mujeres, entender sus orígenes, apreciar evoluciones, conocer sus efectos $\mathrm{y} / \mathrm{o}$ plantear soluciones, para sensibilizar a la población y lograr la comprensión de los problemas de convivencia ciudadana desde la óptica particular de las mujeres, que disminuyan las desigualdades humanas como contribución al desarrollo del país.-

Este trabajo nos permite conocer instrumentos legales internos y convenciones internacionales aprobadas y suscritas por Honduras que mencionamos, que han beneficiado especialmente a las mujeres, que mandan al Estado a diseñar políticas encaminadas a proteger y reducir las brechas que marcan la desigualdad social, y con ello proteger y a las mujeres, fortaleciendo la equidad de género, especialmente en el sector educación como base fundamental para el desarrollo del país.-

Palabras clave: Equidad de género, mujeres, desigualdades sociales, discriminación, sistema educativo.

\section{Abstract}

The reading of this work will take us to identify some existing forms of inequality between men y women, understand its origins, see evolutions, know its effects and/or propose solutions, to sensitize the population and achieve the understanding of the problems of coexistence from the particular perspective of women, that decreases human inequalities as a contribution to the development of the country.

In Honduras in the last decades, they have created institutions and implemented policies that have contributed greatly to reduce educational and employment disparities between women and men. However, although both have access and participate equally in different levels and types of education, barriers and obstacles persist preventing to ensure equality of opportunity in the various stages of society, so there are still some obstacles to overcome in order to put an end to the social inequalities of which they have been direct victims and especially women

Keywords: Equity of genre, Women, Social Inequalities, Discrimination, Educational System 


\section{INTRODUCCIÓN}

El trabajo de Investigación realizado, Ileva como fin recabar información acerca de los diferentes aspectos de lo que es la situación actual de la Equidad de Género en el ámbito educativo de nuestro país; describir el funcionamiento, principales características, sus avances, cifras relevantes y proyectos de cambio dentro del sistema educativo tendientes a fortalecer la equidad.

Dicho trabajo se enmarca en los procesos de cambio que se han venido propiciando por diversas instituciones gubernamentales y no gubernamentales y pudiese servir como punto de partida para elaborar un plan de acción y transversalizar la equidad de género en el Currículo Nacional Básico de Nuestro País.

Esta investigación ha sido realizada a través de una minuciosa recolección de información obtenida de diferentes documentos y artículos relevantes sobre esta temática, así como análisis de documentos y estudios recabados de instituciones dedicadas a tratar situaciones afines al tema.

Con el se espera poder tomar conciencia de que se necesita trabajar aún más en el tema de igualdad especialmente en este ámbito que es el idóneo para empezar a implementar la Educación en Equidad de Género y lograr avances significativos para nuestra sociedad, partiendo de que la desigualdad por razones de género constituye un obstáculo para el derecho a la educación y el desarrollo pleno de las personas.

En este trabajo se trata de identificar la legislación interna e internacional vigente que sirve de marco protector con especialidad de los intereses de las mujeres en Honduras, partiendo de que el siglo pasado fue testigo de la reivindicación de los derechos de las mujeres ante la ley, en la medida en que países de todas las regiones ampliaron el alcance de dichos derechos.- Sin embargo para la mayoría de las mujeres en el mundo, las leyes no se han traducido en mayor igualdad y justicia.

\section{CONCEPTUALIZACIÓN SOBRE GENERO}

El Género son todas las creencias, actitudes, valores, conductas; que la sociedad nos asigna por el hecho de ser hombres o mujeres. Es la construcción social de lo masculino y lo femenino en una cultura, época y sociedad determinada. El género se construye a 
partir del sexo, de lo que cada cultura reconoce como características sexuales y a las cuales les asignan un valor determinado. (Lagarde; 1992)

Frases cotidianas como "¡no seas niña!" o "las mujeres son irracionales" señalan el menosprecio y la desvalorización de lo que son y hacen las mujeres. Establecer algunas funciones como femeninas y otras como masculinas en el ámbito laboral "esto es de mujeres y aquello es de hombres", rechazar a una mujer en un puesto de trabajo por considerar que no es "rentable" si se queda embarazada, son ejemplos de la vida diaria que dan muestra del sexismo y la discriminación por la condición sexual de las personas.

A pesar de que las mujeres son las más afectadas por este tipo de discriminación, también en los hombres hay consecuencias negativas. El sexismo limita en los hombres algunos comportamientos y por lo tanto su desarrollo como persona. Cuando decimos "los niños no lloran" o los "hombres no tienen miedo", estamos inhibiendo sentimientos o comportamientos humanos.

Toda distinción, exclusión o restricción basada en el sexo, que tenga por objeto menoscabar o anular el reconocimiento, goce o ejercicio por parte de la mujer o del hombre independientemente de su estado civil, sobre las bases de la igualdad del hombre y la mujer, de los derechos y las libertades fundamentales en las esferas políticas, económica, social, cultural y civil, o en cualquier otro ámbito es Ilamado discriminación de género. (Montejo, 1992)

Resulta indispensable tener muy claro lo que es la Equidad de Género en la Educación y esta se refiere al Proceso de selección, organización y distribución cultural en el desarrollo curricular, a la igualdad de oportunidades conforme a asignaciones diferenciadas entre hombres y mujeres para que ambos dispongan de las mismas oportunidades para desarrollar su potencial, compartir responsabilidades públicas y privadas en los sistemas sociales, económicos culturales y educativos. (Subirats; 1994)

Así mismo la concientización en género es la toma de conciencia sobre el hecho de que existen diferencias y desigualdades genéricas entre mujeres y hombres, determinadas por la sociedad y la cultura en un tiempo y lugar determinados. Y en la generación del sentido de pertenencia a un género determinado. (Lagarde; 1992)

El sexismo tiene consecuencias para los dos sexos, pero éstas se doblan para las mujeres, porque en una sociedad como la actual, todavía se sitúa a la mujer en una posición de 
inferioridad, mientras que el hombre disfruta de más poder y privilegio en su entorno adjudicándole el papel de dominador sobre la mujer. Los estereotipos sexistas transmiten, mantienen y perpetúan los valores, las creencias y las actitudes que determinan la manera de pensar, actuar y relacionarse de las personas.

La condición de la mujer está influenciada por factores y mecanismos sociales, económicos y culturales que la mantienen en una situación desventajosa y subordinada en relación con el hombre. La forma en que se expresa esta subordinación varía según el contexto histórico cultural. La condición de la mujer, como herramienta conceptual para el análisis de género, se refiere a su estado material, al nivel de satisfacción de sus necesidades prácticas. (Gómariz, 1994)

En este momento es de suma importancia tener en cuenta lo que es la atención a la diversidad en el tema de equidad ya que se refiere a la promoción en los centros educativos, la toma de decisiones curriculares y las prácticas pedagógicas, la igualdad de oportunidades de las mujeres y de los hombres.

Tener claro cada uno de estos conceptos nos ayudarán a tener una mayor comprensión de esta temática que es de gran importancia en estos momentos, ya que la Equidad de Género es una Política Pública y un paso más para mejorar la calidad, cobertura, eficiencia y solidaridad en la Educación.

\section{III.- ANTECEDENTES SOBRE LA EQUIDAD DE GENERO}

La Comisión Interamericana de Mujer (CIM), establecida en 1928, fue el primer órgano intergubernamental en el mundo creado expresamente con el propósito de asegurar el reconocimiento de los derechos civiles, políticos, económicos, sociales y culturales de las mujeres. La CIM está constituida por 34 delegados titulares, uno por cada Estado miembro y se ha convertido en el principal foro generador de políticas hemisféricas para la promoción de los derechos de la mujer y la igualdad de género.

En julio de 1997 el Consejo Económico y Social de las Naciones Unidas (ECOSOC) definió el concepto de la tranversalización de la perspectiva de género en los siguientes términos: "Transversalizar la perspectiva de género es el proceso de valorar las implicaciones que tiene para los hombres y para las mujeres cualquier acción que se planifique, ya se trate de legislación, políticas o programas, en todas las áreas y en todos los niveles.

Es una estrategia para conseguir que las preocupaciones y experiencias de las mujeres, al igual que las de los hombres, sean parte integrante en la elaboración, puesta en marcha, 
control y evaluación de las políticas y de los programas en todas las esferas políticas, económicas y sociales, de manera que las mujeres y los hombres puedan beneficiarse de ellos igualmente y no se perpetúe la desigualdad. El objetivo final de la integración es conseguir la igualdad de los géneros."

La estrategia corporativa del PNUD está diseñada con el fin de integrar en todos los ámbitos de trabajo de la organización el empoderamiento de las mujeres y la equidad. La estrategia Ilama a que esta tarea sea trabajo de todos y no únicamente responsabilidad de algunos especialistas y se apoya en tres pilares:

a. Generación de capacidad tanto a nivel de país como a nivel interno para integrar los desafíos en materia de igualdad de género en todas las áreas temáticas como también en los marcos de cooperación con los países.

b. Provisión de servicios de asesoramiento sobre políticas que promueven la equi dad de género y el empoderamiento de las mujeres y;

c. Apoyo a intervenciones específicas que benefician a las mujeres y extienden modelos innovadores como aquellos desarrollados y probados por el Fondo de Desarrollo de las Naciones Unidas para la Mujer (UNIFEM). (Kemal Dervis, Ad ministrador del PNUD)

En los Objetivos de Desarrollo del Milenio: el camino hacia el futuro (Organización de Naciones Unidas) se definieron como objetivos estratégicos lograr la enseñanza primaria universal y promover la igualdad entre los géneros y la autonomía de las mujeres. Ambos objetivos se complementan, tal y como se planteó en el Informe del Secretario General sobre la aplicación de la Declaración del Milenio, del 31 de julio del 2002, ya que las desigualdades entre los géneros en todos los ciclos de la educación siguen siendo obstáculos al desarrollo económico y a la equidad social.

La creación de un entorno educacional y social en el que se trate en pie de igualdad a las mujeres y los hombres y a las niñas y los niños, en el que se los aliente a alcanzar su pleno potencial, respetando su libertad de pensamiento, conciencia, religión y creencias, y en el que los recursos educacionales promuevan imágenes no estereotipadas de las mujeres y de los hombres, contribuiría eficazmente a eliminar las causas de la discriminación contra las mujeres y las desigualdades entre mujeres y hombres.

Para hacer frente a la desigualdad de acceso a la enseñanza y a las oportunidades educacionales insuficientes, los gobiernos y otros agentes sociales deberían promover una política activa y visible de integración de una perspectiva de género en todas las políticas 
y programas, tal y como se establece en la Cuarta Conferencia Mundial sobre la Mujer a fin de que se analicen, antes de adoptar decisiones, sus posibles efectos en las mujeres y los hombres. Adicionalmente, la convención declara como objetivos estratégicos:

a. Asegurar la igualdad de acceso a la educación.

b. Eliminar el analfabetismo entre las mujeres .

c. Aumentar el acceso de las mujeres a la formación profesional, la ciencia, la tec nología y la educación permanente.

d. Establecer sistemas de educación y capacitación no discriminatorios.

e. Asignar recursos suficientes para las reformas de la educación y vigilar la aplica ción de esas reformas.

f. Promover la educación y capacitación permanentes de las niñas y las mujeres.

El Protocolo adicional a la Convención Americana sobre Derechos Humanos en materia de Derechos Económicos, Sociales y Culturales (Protocolo de San Salvador) y la Convención sobre Todas las Formas de Discriminación contra la Mujer estipulan la eliminación de todo concepto estereotipado de los papeles masculinos y femeninos en todos los niveles y en todas las formas de enseñanza, además, se deben adoptar todas las medidas apropiadas para eliminar la discriminación contra la mujer, a fin de asegurarle la igualdad de derechos con el hombre en la esfera de la educación y en particular para asegurar condiciones de igualdad entre hombres y mujeres. Específicamente, se estipula la necesidad de asegurar:

- Las mismas condiciones de orientación en materia de carreras y capacitación profesional, acceso a los estudios y obtención de diplomas en las instituciones de enseñanza de todas las categorías, tanto en zonas rurales como urbanas; esta igualdad deberá asegurarse en la enseñanza preescolar, general, técnica, profesional y técnica superior, así como en todos los tipos de capacitación profesional

- Acceso a los mismos programas de estudios, a los mismos exámenes, a personal docente del mismo nivel profesional y a locales y equipos escolares de la misma calidad

- La eliminación de todo concepto estereotipado de los papeles masculino y femenino 
en todos los niveles y en todas las formas de enseñanza, mediante el estímulo de la educación mixta y de otros tipos de educación que contribuyan a lograr este objetivo y, en particular, mediante la modificación de los libros y programas escolares y la adaptación de los métodos de enseñanza

- Las mismas oportunidades para la obtención de becas y otras subvenciones para cursar estudios

- Las mismas oportunidades de acceso a los programas de educación permanente, incluidos los programas de alfabetización funcional y de adultos, con miras en particular a reducir lo antes posible toda diferencia de conocimientos que exista entre hombres y mujeres

- La reducción de la tasa de abandono femenino de los estudios y la organización de programas para aquellas jóvenes y mujeres que hayan dejado los estudios prematuramente

- Las mismas oportunidades para participar activamente en el deporte y la educación física

- Acceso al material informativo específico que contribuya a asegurar la salud y el bienestar de la familia, incluida la información y el asesoramiento sobre planificación de la familia

La Declaración mundial sobre la educación superior en el siglo XXI: visión y acción (Tailandia, 1990), reconoce la necesidad de ofrecer a las generaciones presentes y venideras una visión ampliada de la educación básica y un renovado compromiso en favor de ella, para hacer frente a la amplitud y a la complejidad del desafío, esto comprende:

- Universalizar el acceso a la educación y fomentar la equidad

- Prestar atención prioritaria al aprendizaje

- Ampliar los medios y el alcance de la educación básica

- Mejorar el ambiente para el aprendizaje

- Fortalecer concertación de acciones.

Adicionalmente, la Convención Internacional sobre la Eliminación de todas las formas de Discriminación Racial establece en su artículo 7 que "Los Estados partes se compro- 
meten a tomar medidas inmediatas y eficaces, especialmente en las esferas de la enseñanza, (...) para combatir los prejuicios que conduzcan a la discriminación racial y para promover la comprensión, la tolerancia y la amistad entre las naciones y los diversos grupos raciales o étnicos (...)".

El 8 de marzo del año 2011 se cumplieron los primeros 100 años del Día Internacional de la Mujer, en el que se celebraron también los grandes avances que en este siglo se han logrado en la búsqueda de la igualdad de derechos; aunque todavía quedad muchísimo camino por recorrer para que se reconozca de forma plena el papel de las mujeres en la historia humana y la necesidad de ponerle fin a toda forma de discriminación.

En la Organización de las Naciones Unidas recién empiezo a funcionar la entidad de la ONU para la Igualdad de Género y el Empoderamiento de la Mujer (ONU Mujeres), un organismo que tiene como propósito acelerar los esfuerzos internacionales para alcanzar la igualdad de género y cuya directora ejecutiva es la ex presidenta chilena, la socialista Michelle Bachelet, quien sostiene que "La fuerza, el trabajo y la inteligencia de las mujeres son la gran fuente menos explotada de la humanidad". Y es que al igual que la brecha científica y tecnológica es la principal diferencia entre los países desarrollados y los subdesarrollados, la igualdad de género también pasa porque no se continúe obstruyendo el acceso de las niñas y las mujeres al conocimiento y a la tecnología.

La igualdad de género es una cuestión de justicia, de ética, de humanismo; pero también de conveniencia para la humanidad entera. Y es que si ya de por si la presencia de mujeres en los órganos de poder de las naciones, organismos internacionales, en el sector empresarial, etc. aporta una dinámica distinta, positiva, cuando ese empoderamiento de la mujer llegue a las zonas más vulnerables de los países pobres se producirá un impacto fuerte y directo en asuntos de importancia clave como la lucha contra el hambre, la pobreza y la emigración. (Editorial Diario El Heraldo; 8 de Marzo2011)

Para el Instituto Nacional de la Mujer (INAM) la relación actual del tema de Género en Educación con la Secretaría de Educación ha sido abierta y horizontal.

Desde al año 2002 a la fecha y como productos de las investigaciones realizadas por el INAM en coordinación con la Secretaría de Educación en temas como reconocimiento de la diversidad cultural y equidad de género en el nivel básico de la educación, la valoración temática del enfoque de género en el Currículo Nacional Básico y un estudio sobre el sistema de gestión docente en Honduras, de manera constructivista y participativa se construye la propuesta de apoyo a la cultura de equidad en la educación pre-básica, 
básica y media partiendo del interés conjunto en:

a. Impulsar una política de equidad de género en la transformación educativa nacional.

b. Contribuir a resolver problemas de calidad, equidad, eficiencia y cobertura del Sistema Educativo.

c. Reconocer que la coeducación es necesaria pero no suficiente para el logro del principio de igualdad de oportunidades.

Se debe tomar muy en cuenta que el Currículo Nacional Básico construye su plataforma política desde la correlación intersectorial, la atención a la diversidad, la equidad de género, la reducción de la pobreza, el desarrollo profesional docente, y el logro de altos niveles de desempeño acción política que se fortalece con la propuesta de apoyo a la cultura de equidad en la educación. Esta propuesta engloba dentro de su visión política, técnica y metodológica temas como:

1. La inclusión y sus contextos para el desarrollo humano sostenible desde una visión de país.

2. Inclusión y cohesión social

3. Autonomía económica

4. Gobernabilidad democrática

A nivel mundial el PNUD promueve la equidad de género a través de la transversalización de la perspectiva de género.

\section{IV.- AVANCES SOBRE EQUIDAD EN EL MARCO JURÍDICO NACIONAL E INTERNA- CIONAL EN BENEFICIO A LAS MUJERES}

La igualdad de género de las personas es un principio constitucional que aparece por primera vez con claridad en la Constitución Política de 1831, la cual expresa en su articulo 11 "todos los hondureños son libres ante la ley.....". Sin embargo, en nuestro país, la tradición jurídica colonialista no permitió extender el alcance de este principio, sobre todo para efectos civiles y políticos, sino hasta la Constitución de 1957.- 
La igualdad ante la ley, en el caso de la mujer, tiene en realidad reciente protección y actualmente el reconocimiento de la igualdad jurídica de las mujeres frente a los hombres es una realidad. En la Constitución de la Republica vigente, creada mediante Decreto Número 131 del 11 de Enero de 1982, es en el artículo 60 que garantiza la igualdad de derechos que tenemos los hondureños cuando manda que "Todos los hondureños nacen libres e iguales en derechos....Todos los hombres son iguales ante la ley..."

El contexto nacional en materia de Derecho y Equidad de Género se caracteriza por considerables avances en la adecuación del marco jurídico-legal, así como por la progresiva consolidación de la institucionalidad de género en el Estado de Honduras. La legislación nacional en materia de promoción y protección de los Derechos Humanos de las mujeres y eliminación de todas las formas de discriminación de las mujeres, se encuentra plasmada en un conjunto de leyes y políticas tales como:

1.- La Constitutcion de la Republica (Decreto No. 131 del 1982), en su artículo número 60 garantiza la igualdad de derechos de todos los hondureños y declara punible la discriminación hacia las personas.

2.- La Ley Contra la Violencia Doméstica (1997) y sus reformas (2005), promueve un cambio en los valores vigentes y establece la obligación del Estado de ejecutar políticas públicas que garanticen la eliminación de la violencia doméstica y la protección de la familia.Esta ley fue reformada casi en su totalidad en el año 2005, y con estas reformas se introdujeron significativos avances en la ley, entre ellos la sanción del acto que motiva la denuncia de violencia doméstica, la introducción de mecanismos de coordinación y seguimiento, el aumento del período de imposición de las sanciones y otros más.

3.- El Código Penal en las reformas incluidas en 1996, 1997 y 2005 coadyuvan a la garantía del derecho a la paz y a una vida libre de violencia.

4.- La Ley de Igualdad de Oportunidades para la Mujer (LIOM - 2000) y su reglamento (2008) establece la obligación del Estado de promover la igualdad y la equidad de género, proteger los derechos de las mujeres en los diferentes ámbitos: familia, salud, educación, cultura, trabajo y seguridad social, tenencia de la tierra, acceso al crédito, a la vivienda, participación política y en la toma de decisiones en las estructuras de poder.

5.- La Ley del Instituto Nacional de la Mujer, INAM, aprobada mediante el Decreto Legislativo 232-98 del 11 de febrero de 1999. 
6.-La Ley Electoral y de las Organizaciones Políticas (LEOP) entró en vigencia en 2004 y en su capítulo II habla de igualdad de oportunidades políticas. Referente a las mujeres sólo tiene tres artículos, en el artículo 103 establece que el Estado debe garantizar la democracia participativa y el ejercicio pleno de los derechos ciudadanos tanto a hombres como a mujeres, en igualdad de oportunidades, y que los partidos políticos deben crear las condiciones materiales que permitan la incorporación real de las mujeres en todos los aspectos de la vida partidaria.

El artículo 104 se refiere a la garantía de no discriminación y establece la obligación del Estado, por medio del Tribunal Supremo Electoral, de vigilar que en las estructuras de gobierno de los partidos políticos y en las candidaturas a cargo de elección popular, no exista discriminación por razón de género, credo, raza, religión y cualquier otra forma de discriminación. Asimismo menciona que los partidos políticos aprobarán internamente, con la participación de las mujeres, una política de equidad de género, cuyo cumplimiento será supervisado por el Tribunal Supremo Electoral; además, obliga a los partidos políticos a presentar al Tribunal un informe del cumplimiento de la Política de Equidad de Género, seis (6) meses antes de la convocatoria a las elecciones internas y primarias.

En el artículo 105 habla de la distribución equitativa en los cargos de elección popular y menciona que para lograr la participación efectiva de la mujer, se establece una base de treinta por ciento (30\%) como mínimo, aplicable a los cargos de dirección de los Partidos Políticos, Diputados propietarios y suplentes al Congreso Nacional, al Parlamento Centroamericano, Alcaldes, Vice Alcaldes y Regidores.

Otras leyes vigentes tales como el Código de Familia, el Código del Trabajo, la Ley de $\mathrm{VIH}$ y Sida; incluyen acciones afirmativas para la garantía del ejercicio de los derechos de las mujeres en sus ámbitos específicos.

El Estado ha ratificado un conjunto de instrumentos internacionales relacionados con la igualdad y equidad de género y la protección a las mujeres, las adolescentes y las niñas, asumiendo el compromiso de establecer medidas de carácter obligatorio para el avance y protección de sus derechos. Cabe destacar que los Tratados y Convenciones Internacionales, una vez ratificados por el Estado Hondureño, forman parte del derecho interno.

Desde 1975, declarado como Año Internacional de la Mujer, se han desarrollado conferencias mundiales y promovido convenciones internacionales impulsadas por las Naciones Unidas, con el objetivo de mejorar la situación de las mujeres. La primera conferencia celebrada en México (1975) proclamó que la siguiente década se denominaría: 
El Decenio de las Naciones Unidas para la Mujer (1976-1985). En ella se aprobó la declaración por la Igualdad de la Mujer y su Contribución al Desarrollo y la Paz. Y se definió un plan de acción mundial para la consecución de los siguientes objetivos: la igualdad plena de género y la eliminación de la discriminación por motivos de género, la integración y plena participación de la mujer en el desarrollo, una contribución cada vez mayor de la mujer al fortalecimiento de la paz mundial.En el plan de acción se estableció un mínimo de metas, para alcanzarlas en 1980, que se centraban en garantizar el acceso equitativo de la mujer a los recursos, como la educación, las oportunidades de empleo, la participación política, los servicios de salud, la vivienda, la nutrición y la planificación de la familia.

La Segunda Conferencia Mundial sobre la Mujer se realizó en Nairobi, África, en 1985. Tenía como objetivo evaluar los avances y los obstáculos enfrentados durante el Decenio de las Naciones Unidas para la Mujer. Se aprobó por consenso el documento denominado "Las Estrategias de Nairobi" orientadas hacia el futuro para el adelanto de las mujeres hasta el año 2000, que constituyen iniciativas a adoptar a nivel nacional, regional e internacional para promover el reconocimiento social del papel de las mujeres y el ejercicio de sus derechos humanos. En esta conferencia la evaluación fue muy crítica, los informes estadísticos reunidos por la ONU revelaban que las mejoras en la situación jurídica y social de la mujer y los esfuerzos para reducir la discriminación habían beneficiado únicamente a una pequeña minoría de mujeres y muy marginales.

En septiembre de 1994, en El Cairo, se Ilevó a cabo la Conferencia Internacional sobre la Población y el Desarrollo (CIPD). En esta conferencia se acordó que la población y el desarrollo están indisolublemente unidos; y que es necesario dotar de mayor poder a la mujer y tomar en cuenta las necesidades de la gente en lo relativo a educación y salud, incluyendo la salud reproductiva, para el avance individual y el desarrollo balanceado. Avanzar en la equidad de género, eliminar la violencia contra las mujeres y asegurar la habilidad de las mujeres de controlar su propia fertilidad eran pilares de las políticas de población y desarrollo. Las metas concretas fueron: proveer educación universal y cuidado a la salud reproductiva que incluya la planeación familiar y la reducción dela mortalidad maternal e infantil.

Luego, en 1995, se llevó a cabo en Beijing la IV Conferencia Mundial sobre la Mujer, que abre una nueva etapa en la lucha por eliminar la discriminación de las mujeres.

Se logró introducir el concepto de género y desde allí dar una nueva mirada a las estructuras de las sociedades, y a todas las relaciones entre los hombres y las mujeres. 
En Beijing se consideró que la problemática de las mujeres afecta a la sociedad en su conjunto y se considera, por primera vez, que su tratamiento no puede ser sectorial y tiene que integrarse en el conjunto de políticas. Se aprobó por unanimidad la Declaración y la Plataforma de Acción de Beijing, que era en esencia un programa para la potenciación del papel de la mujer y representa un momento decisivo para el adelanto de la mujer en el siglo XXI.

La convención sobre la Eliminación de todas las Formas de Discriminación contra la Mujer, conocida como CEDAW, por sus siglas en inglés, ratificada por Honduras en 1981, protege el derecho de las mujeres a la no discriminación (Art.1); a la libertad de elegir empleo, a salario igual por trabajo igual, a la estabilidad laboral en estado de embarazo y licencia con remuneración; y el derecho al cuidado infantil.

En la CEDAW las mujeres lograron ser reconocidas como humanas, como personas con dignidad y capacidad para decidir sobre su desarrollo en igualdad y libertad.

Lograron no ser consideradas un bien común de la sociedad (las vidas de las mujeres no pueden ser controladas por otras personas); no ser consideradas como objeto que puede utilizarse para dar placer sexual; el derecho a vivir una vida libre sin violencia, sin agresiones contra la imagen femenina que transmiten los medios de comunicación, sin maltrato físico, emocional, sexual o intelectual, en la casa, en el trabajo o en cualquier lugar; y a gozar de igualdad de derechos en el acceso a recursos productivos como la tierra, el crédito, la asistencia técnica y capacitación laboral; y el derecho a la vida.

La Convención Interamericana para Prevenir, Sancionar y Erradicar la Violencia Contra la Mujer, conocida como Belem Do Pará, aprobada en la OEA en 1995, es un instrumento muy importante a nivel internacional para la defensa de los derechos de las mujeres y por la equidad entre hombres y mujeres. Esta convención reconoce que existe una discriminación institucionalizada que condiciona el desarrollo y la condición jurídica y social de las mujeres en el mundo, no sólo la violencia que se da en el ámbito privado sino también aquella que se da en el ámbito público, la violencia que sea perpetuada por cualquier persona en la comunidad comprendiendo el acoso sexual en el trabajo, la que sea perpetuada o tolerada por los agentes del Estado, donde quiera que ocurra.

El Estado de Honduras al firmar esta convención se ha obligado a llevar a cabo lo siguiente: Abstenerse de cualquier acción o práctica de violencia contra la mujer y velar porque las autoridades, sus funcionarios personales y agentes e instituciones se comporten de conformidad con esta obligación; actuar con la debida diligencia para prevenir, investi- 
gar y sancionar la violencia contra la mujer; adoptar medidas jurídicas para conminar al agresor a abstenerse de hostigar, intimidar, amenazar, dañar o poner en peligro la vida de la mujer de cualquier forma que atente contra su integridad o perjudique su propiedad; tomar todas las medidas apropiadas para modificar prácticas legales o costumbres que respalden la persistencia o la tolerancia de la violencia contra la mujer; establecer los mecanismos judiciales y administrativos necesarios para asegurar que la mujer objeto de violencia tenga acceso efectivo a resarcimiento, reparación del daño, u otros medios de compensación justos y eficaces.

Las convenciones internacionales, una vez firmadas y ratificadas por un gobierno, tienen carácter vinculante, es decir que los Estados Parte se comprometen a cumplir las disposiciones contenidas en las convenciones y, además, a incluirlas en las leyes internas, de ahí la razón por la que es importante estudiar y reconocer la importancia de las convenciones firmadas por nuestro país, entre ellas están dos relacionadas específicamente con las mujeres.

\section{A.- Convenios Internacionales de Derechos Humanos ratificados por el Estado de Honduras}

Los instrumentos internacionales de Derechos Humanos ratificados por el Estado de Honduras desde hace varias décadas, contribuyen sin lugar a dudas a fortalecer el marco legal e institucional sobre diferentes temas, entre los Convenios más importantes, destacan:

\section{Nombre del Instrumento Internacional}

Pacto Internacional de Derechos Civiles y Políticos

Carta de la Organización de los Estados Americanos

Convención sobre los Derechos del Niño

Declaración Americana de los Derechos y Deberes del Hombre

Convención Americana sobre Derechos Humanos o Pacto de San José

Convención Internacional sobre la Eliminación de todas las formas de Discriminación contra la Mujer

Convención Interamericana para Prevenir Sancionar y Erradicar la Violencia contra la Mujer Convenio para la represión de la Trata de Personas y de la Explotación de la Prostitución Ajena

Protocolo facultativo para la Convención de Naciones Unidas sobre derechos del niño relativo a la venta de niños, la prostitución infantil y la utilización de niños en la pornografía

\section{Fecha de Ratificación}

18-06-1995

02-07-1950

$10-1990$

23-06-1949

05-09-1977

02-04-2002

25-04-1995

15-06-1993

08-05-2002 
A pesar de todas estas regulaciones y compromisos del Estado por el derecho a la educación, podemos decir que éste se incumple desde el punto de vista del acceso, porque no hay un acceso universal y gratuito como se contempla en la declaración de los derechos de la niñez, pero también se incumple en el aspecto de calidad y equidad, puesto que la educación que se recibe en los centros educativos tiene muchas limitantes cualitativas

\section{V.- LA EDUCACION Y LA EQUIDAD DE GENERO}

La Declaración Universal de Derechos Humanos, en particular, el párrafo 1 de su Artículo 26, declara que: "Toda persona tiene derecho a la educación, debe ser gratuita, al menos en lo concerniente a la instrucción elemental y fundamental. La instrucción elemental será obligatoria, la instrucción técnica y profesional habrá de ser generalizada, en función de los méritos respectivos, y que el acceso a los estudios superiores será igual para todos".

La educación es un derecho humano, tal y como se declara en la Cuarta Conferencia Mundial sobre la Mujer (Beijing 1995); constituye un instrumento indispensable para lograr los objetivos de la igualdad, el desarrollo y la paz. La educación no discriminatoria beneficia tanto a las niñas como a los niños y, de esa manera, conduce en última instancia a relaciones más igualitarias entre mujeres y hombres. La igualdad de acceso a la educación y la obtención de educación son necesarias para que más mujeres se conviertan en agentes de cambio. La alfabetización de la mujer es importante para mejorar la salud, la nutrición y la educación en la familia, así como para habilitar a la mujer para participar en la adopción de decisiones en la sociedad. Ha quedado demostrado que la inversión en la educación y la capacitación formal y no formal de las niñas y las mujeres, tiene un rendimiento social y económico excepcionalmente alto, es uno de los mejores medios de lograr un desarrollo sostenible y un crecimiento económico a la vez sostenido y sostenible.

En la inauguración del Foro Mundial de Educación celebrado en Dakar en el año 2000, el Sr. Kofi Annan, Secretario General de las Naciones Unidas, reconoce que en el comienzo del siglo XXI hay algunos logros que celebrar a nivel mundial.

Los niveles educativos de numerosos países se han elevado, el porcentaje de personas adultas analfabetas ha disminuido, la explosión de innovaciones tecnológicas ha aportado nuevas posibilidades de aprendizaje a millones de personas. Sin embargo, al menos 880 millones de personas adultas, en su mayoría mujeres, son analfabetas. Doscientos 
cincuenta mil millones de niños trabajan en condiciones a menudo peligrosas o insalubres. Y, según estimaciones moderadas, más de 110 millones de niños en edad escolar no están escolarizados.

En esa misma conferencia, Kofi Annan hizo énfasis en la situación educativa de las niñas: "En términos tanto de moralidad como de mortalidad, la negación de los derechos de las niñas empiezan en la primera infancia. Cuando se debe elegir entre educar a un niño o a una niña, es más probable que ésta última permanezca en el hogar. Cuando es necesario completar los ingresos de la familia, se suele enviar a las niñas a trabajar. Aún cuando las niñas vayan a la escuela, a menudo deben ocuparse de quehaceres domésticos a expensas de sus tareas escolares. Cuando quedan embarazadas, las políticas educativas las obligan abandonar las escuelas. Cuando los padres piensan en el futuro de sus hijas perciben generalmente, la educación no como una ayuda, sino como un obstáculo para el éxito del casamiento y la maternidad. Y si se produce una tragedia, ya sea en forma de enfermedad o de conflicto, desplazamiento o dificultades materiales, las mujeres son las que más probablemente asumirán la carga de mantener a la familia y el hogar."

"Se dice a menudo que la educación contribuye a la emancipación de las niñas al fortalecer su confianza en sí mismas y permitirles adoptar decisiones informadas acerca de sus vidas." "Para la mayoría de las niñas del mundo, se trata de algo mucho más fundamental. Se trata de huir de la trampa del trabajo infantil, de los riesgos de iniciar el trabajo de parto siendo una niña aún, de administrar los embarazos de modo que no amenacen la salud, los medios de subsistencia o incluso la vida, de asegurar que sus hijos, a su vez, tengan garantizados su derecho a la educación. Se trata de poder ganar dinero, cuando las mujeres antes no lo hacían; protegerse a sí misma contra la violencia y gozar de los derechos que las mujeres nunca había tenido en tiempo pasados, de participar en la adopción de decisiones económicas y políticas y por último de educar a los hijos para que hagan lo mismo, y sus hijos también. Se trata de poner término a la espiral de pobreza e impotencia que anteriormente parecía no tener fin." (Informe Final. Foro Mundial sobre la Educación, Dakar, 2000).-

Asímismo en este Foro los Estados parte se comprometieron a eliminar las disparidades de género en la enseñanza primaria y secundaria de aquí al año 2005 y lograr la igualdad de género en el año 2015, cuidando de garantizar a las niñas y adolescentes un acceso equitativo, real y sin restricciones a una educación de base de calidad. También plantearon la necesidad de poner en marcha estrategias integradas para la igualdad de los géneros en educación, que reconozcan la necesidad de un cambio de actitudes, de valores y de prácticas. 


\section{VI.- LAS MUJERES Y LA EDUCACIÓN EN HONDURAS}

En Honduras en las últimas décadas, se ha reducido la brecha educativa entre mujeres y hombres. Sin embargo, aunque ambos acceden y participan de manera igualitaria en los distintos niveles y modalidades de la educación, persisten barreras y obstáculos que impiden garantizar la igualdad de oportunidades en los diversos escenarios de la sociedad.

En todos los niveles, según datos estadísticos proporcionados por la Secretaría de Educación, el porcentaje de aplazamiento y reprobación respecto a la matrícula inicial por sexo es superior en los hombres que en las mujeres.

El rendimiento mejor es el de las mujeres y su mayor permanencia en el sistema educativo con relación a los hombres parece reflejar una conducta de género, en el sentido de que la socialización primaria a que se somete a las mujeres, cargada de responsabilidades y de valores como el orden, la disciplina y el "recogimiento" estarían dando como resultado un mayor compromiso con la escuela. Este a su vez estaría actuando como un factor positivo en tanto da réditos a las mujeres y sería deseable propiciar en el ámbito familiar y escolar un efecto emulador en los hombres, que los lleve a un comportamiento más responsable y comprometido con los estudios, similar al de las mujeres.

A pesar de que las cifras obtenidas y analizadas reflejan una participación cuantitativa casi paritaria entre mujeres y hombres en los distintos niveles del sistema educativo, esta igualdad formal no está acompañada de una igualdad real, dado que los procesos de enseñanza y aprendizaje se continúan desarrollan en un contexto de discriminación y desventaja hacia las mujeres, que se manifiesta en sus diversos componentes y que atenta contra la equidad entre los géneros.

El sistema educativo además de transmitir, por medio del currículum oficial, las nociones culturales relativas al género aceptadas socialmente, también deberían transferir un cúmulo de pautas de comportamiento no explícitas, que influyen en la construcción de la identidad de niñas y de niños, en su confianza y autovaloración, así como en sus motivaciones y expectativas de vida. A este proceso de transmisión velada de las concepciones de género se le denomina "el currículum oculto" o "la pedagogía invisible de género".

Las normas que rigen el funcionamiento escolar, las costumbres, la distribución del aula y de los lugares de recreo, los juegos, la forma de impartir educación física, los conte- 
nidos de los programas, el material didáctico, la organización administrativa escolar, la actitud de los y las docentes hacia sus estudiantes, así como el modelo que representan las figuras de autoridad dentro de la escuela, constituyen algunos de los mecanismos para transmitir y reforzar los valores, normas y concepciones acerca de cómo deben ser y actuar los hombres y las mujeres. Esto influye en la configuración y perpetuidad de los papeles y estereotipos de género, e incide en el desarrollo de relaciones equitativas entre los sexos.

Los datos nos reflejan que a pesar de que las mujeres abandonan sus estudios en menor medida que los hombres y obtienen un elevado rendimiento académico, esto no ha representado en la mayoría de los casos, una mayor y mejor inserción en la vida social, política y económica del país. En consecuencia, una alta calificación de la población femenina no ha conducido a un mejoramiento de su calidad de vida.

Esto parece indicar que nuestro sistema educativo no ha dado respuesta efectiva a un sector amplio de mujeres, para quienes la educación ha dejado de ser un instrumento de movilidad social, en el sentido de asegurar acceso al trabajo y a una remuneración justa, que concuerde con el nivel de formación alcanzado por esta población. La preparación superior que tienen las mujeres respecto de los hombres no llega a traducirse, en la mayoría de los casos, en mejores condiciones laborales.

Según el Artículo Informe Rendimiento Académico, las Niñas tienen mejor desempeño que los niños en todos los niveles (Diario El Heraldo 2011, 11 de Mayo): "Las niñas superan levemente entre primer y sexto grado a los niños en todos los niveles educativos según el informe nacional de rendimiento escolar en español y matemáticas de 2010.- A pesar que las niñas superan levemente a los niños, se puede observar que la mayoría de estudiantes se encuentra en el nivel de "debe mejorar", excepto los alumnos que cursaron quinto grado en el 2010, cuyo desempeño se encuentra en el nivel satisfactorio. Tanto en primer como en sexto grado las niñas obtienen mejores resultados que los niños"

Esto nos demuestra que efectivamente, las mujeres tienen la oportunidad de salir adelante deben tomarla y realizar su mejor esfuerzo para cumplir con sus metas.

La educación como derecho humano y elemento indispensable para el progreso económico y social y debe reconocerse que el acceso pleno y las condiciones de igualdad en educación es un requisito fundamental para la potenciación de la mujer, y un instrumento fundamental para lograr los objetivos de igualdad de género, desarrollo y paz.

Pese a que se ha informado de progresos, persisten grandes diferencias entre el hombre y 
la mujer en lo que respecta a su acceso a la educación. La eliminación de la alta tasa de analfabetismo entre las mujeres y las niñas sigue siendo una urgente tarea a nivel mundial. De acuerdo con el Instituto de Estadística de la UNESCO, se calcula que en la actualidad dos terceras partes de los 875 millones de adultos analfabetos en el mundo son mujeres. En Asia meridional, casi tres de cada cinco mujeres son analfabetas, y se calcula que todavía la mitad de todas las mujeres en África y la región árabe son analfabetas.

Las disparidades por motivos de género en las tasas de alfabetización son menos pronunciadas entre los adultos jóvenes. En la actualidad apenas hay diferencia, o no hay diferencia ninguna, en las tasas de alfabetización de las personas de 15 a 24 años de edad en varias regiones del mundo, como Europa, América del Norte, América Latina y el Caribe, y Asia oriental y Oceanía.

En la actualidad la educación de Honduras presenta varias circunstancias que colocan a los niños y las niñas en desventaja de poder acceder al sistema educativo y de tener las mismas oportunidades para el desarrollo de sus habilidades, ingenio y creatividad. Es así que la cobertura en todos los niveles es insuficiente, además de presentar diferentes estándares de calidad.

Se han disminuido sustancialmente las tasas de analfabetismo tanto para hombres como para mujeres, aunque casi dos de cada 10 personas mayores de 9 años son analfabetas en el país con un alto porcentaje de estas personas con edades mayores a los 35 años. Aunque a nivel general, una de cada cinco personas no sabe leer y escribir, esta proporción se reduce a uno de cada 10 personas en el área rural y a 5 de cada 10 personas en el caso de mayores de 60 años.

El ritmo de la reducción del analfabetismo es más acelerado para mujeres que para hombres, probablemente por el creciente aumento de la matrícula femenina en los diferentes niveles educativos. Ello no obstante, no ha incidido en una mejoría sustancial en el nivel y calidad de vida de las mujeres.

Según el Censo de Población y Vivienda de 2011 estadísticas cuyos cambios al año 2013 han sido mínimos, de cada 100 personas de 10 años y mas que saben leer y escribir 49 son hombres y 51 son mujeres, similar a la proporción de la población según sexo. Desde otra perspectiva, las tasas de alfabetización por sexo, definidas como la población mayor de 15 años que sabe leer y escribir, da cuenta que la brecha paso de ser negativa para las mujeres a cerrarse e incluso superar en casi un punto a los hombres. A lo largo 
del tiempo ha habido más hombres alfabetos que mujeres, sin embargo al revisar el comportamiento de acuerdo al censo 2013, se observa mayor incorporación y acceso de las mujeres en educación.

\section{A.- LA DESIGUALDAD DE GÉNERO EN CIFRAS.}

Honduras según el INE al año 2013, cuenta con una población de 8,555.1 habitantes, de los cuales 4,213.7 son hombres y el 4,341.4 son mujeres, siendo mayoritarias las mujeres. Del total se estima que el $78.6 \%$ de los hondureños están en edad de trabajar y que la población económicamente activa es del $42 \%$.

Los datos revelan que en Honduras, de acuerdo al promedio de años de estudio, la mujer tiene un mayor nivel de escolaridad que los hombres. De acuerdo al estudio la escolaridad de las mujeres es de 6.4 años y la de los hombres de 6.0 años.

El estudio también refiere que el $71 \%$ de los hombres cuentan con un empleo, mientras que el $29 \%$ restantes están en condiciones de desempleo. En lo que respecta a la mujer, el informe establece que el $37.4 \%$ están empleadas y que el $62.6 \%$ no cuenta con una fuente de trabajo. (Fuente INE, 2011)

No obstante el rezago que ha tenido el sexo femenino en nuestra sociedad, algunas cifras en educación superior son demostrativas de la supremacía de las mismas a accesar a este nivel educativo, como lo demuestra las estadísticas del año 2013 de matrícula, tanto en la UNAH y las Universidades Privadas de Honduras, como se señala a continuación:

a. Matrícula por sexo en Universidades Publicas:

- Masculino - 41,147 (39.5\%)

- Femenino $-63,147 \quad(60.5 \%)$

b. Matrícula por sexo en Universidades Privadas:

- Masculino - 31,120 (45.5\%)

- Femenino - 37,123 (52.6\%)

c. Matrícula de la Universidad Nacional Autónoma de Honduras:

- Masculino - 28,786 (40.1\%)

- Femenino - 42,979 (59.9\%)

d. En cuanto a los Docentes en la UNAH predomina el sexo masculino:

- Masculino - 4,985 (58\%)

- Femenino - 3,608 (42\%) 


\section{1.- Desigualdades hacia las mujeres en la educación}

Honduras se ubica en el escenario de mayor desigualdad, pobreza y exclusión, no solo por las consecuencias de su propia historia socio-política, sino, además, por la posición desde donde nos insertamos en el mercado global.

Este contexto no configura el mejor escenario para lograr la igualdad de género.

El acceso a la educación primaria y secundaria no significa que la escuela haya dejado de ser uno de los espacios de socialización y reproducción de las desigualdades por excelencia. Tampoco significa que ello se ha traducido en una mejor inserción en el mercado de trabajo ni en una reducción de la brecha salarial entre mujeres y hombres, que por cierto, se amplía a mayores años de estudio.

La Comisión Interamericana de Derechos Humanos de la Organización de los Estados Americanos ha interpretado que las medidas de acción positiva son compatibles con los principios de igualdad y de no discriminación. En el caso de Honduras, algunas medidas de acción positiva son:

a. Los mecanismos para el avance de las mujeres (INAM, OMM, Oficinas de Género dentro de las Secretarías de Estado, la Fiscalía General de la Mujer)

b. Ley de Igualdad de Oportunidades para la Mujer

c. La Política Nacional de Igualdad de Oportunidades de Honduras 2002-2007, instrumento oficializado como Política de Estado en noviembre del 2002 según Decreto Ejecutivo No. 015-2002, reafirmando el compromiso con el avance de las mujeres.

No obstante, la oficialización por sí sola no garantiza la ejecución si no se contempla la equidad en las acciones, en la estructura del gasto público y en los niveles de asignación presupuestaria necesaria para su implementación desde las diferentes instituciones y secretarias relacionadas con esta política. (Regina Fonseca, 2009).

Honduras sigue siendo un país de corte machista, con escasas oportunidades y hace que las mujeres sean víctimas de exclusión en la sociedad y en el ambiente donde se desenvuelven. Jennifer Vaughan, directora de Plan Honduras, dijo que en el país, al igual que en el resto de los países latinoamericanos, las niñas sufren una doble discriminación 
por ser jóvenes y por ser mujeres, las que son sacadas de la escuela, casadas a temprana edad y son mas sujetas a la violencia; también menciono la importancia de transformar el curriculum escolar para restar los estereotipos, apoyar la participación de las niñas y los niños en la creación de políticas públicas para optimizar la igualdad de género. (Acosta; 2011)

\section{2.- Desigualdades hacia las mujeres en el ambito laboral}

De acuerdo a los resultados a nivel nacional las mujeres sin ningún nivel de instrucción trabajan cuatro horas en labores domesticas, mientras que los hombres apenas 1.29 hrs. Los hombres con mayor nivel de instrucción dedican mayor tiempo al trabajo doméstico.

La participación de las mujeres en el mercado laboral nacional, es cada vez mayor, sin embargo, esto no ha implicado una disminución de la carga y el tiempo dedicado al trabajo doméstico. El INE encontró que en Honduras, la participación de las mujeres en tales labores, en el área urbana, supera el $86 \%$ por ciento, incrementándose a $91.36 \%$ en el área rural.

Según el PNUD, Honduras tiene en su legislación tratados internacionales que mandan a los Estados a diseñar políticas para ir reduciendo estas brechas que marcan la desigualdad social. Los estudios deben servir para que el Estado diseñe políticas que permitan el cambio de roles y que pongan a las mujeres en iguales condiciones que los hombres tanto en trabajo, educación e ingreso económico ha señalado este organismo internacional.

\section{B.- EMPODERAMIENTO DE LAS MUJERES}

El empoderamiento del papel de la mujer y la igualdad entre la mujer y el hombre son condiciones indispensables para lograr la seguridad política, social, económica, cultural y ecológica entre todos los pueblos. (Declaración y Plataforma de Beijing, 1995)

El Estado Hondureño considera fundamental para el desarrollo del país, que mujeres y hombres tomen conciencia de sus derechos y se reconozcan plenamente como sujetas y sujetos de derechos y responsabilidades. El empoderamiento de las mujeres hondureñas implica por lo tanto el desarrollo de capacidades, destrezas y habilidades para participar activamente en los procesos económicos, sociales y políticos del país. Esa capacidad equivale a mayor poder de decisión y autonomía política, social, económica, física y cultural.

Es un proceso por medio del cual las mujeres adquieren la capacidad de construir su pro- 
pio plan de vida, establecen con claridad y determinación la forma de relacionamiento con su familia, pareja, colegas, socias y socios, jefes y jefas y frente a las instituciones sociales, convirtiéndose en agentes de desarrollo.

Es vital fomentar la autoconfianza de las mujeres, la seguridad en sí mismas, el poder para tomar decisiones sobre sus vidas, de manera individual y colectiva, para alcanzar objetivos como la equidad de género. Las mujeres empoderadas estarán en condiciones de superar la subordinación, ya que han vivido en un proceso de reconocimiento de la ideología de dominación masculina que reproduce patrones socioculturales de discriminación y exclusión hacia ellas.

\section{1.- Principales logros de las mujeres en educación}

- Disminución de los índices de analfabetismo entre las mujeres.

- Ampliación de la cobertura regional de la educación de niñas y jóvenes.

- Implementación del enfoque de género en el currículo nacional básico, en todos los niveles del sistema educativo y en los textos de educación.

- Implementación del enfoque de desarrollo de habilidades en programas de alfabetización radial.

- Aprobación de normativa jurídica y materiales de apoyo y guías educativas para la educación con enfoque de género.

- Realización de campañas comunicacionales sobre la igualdad de género que aportaron al posicionamiento del tema y a la sensibilización de la población.

- Incorporación del enfoque de género en los registros de información del sistema educativo nacional.

- Organización de la Red de Comunicadores Sociales por una Cultura de Equidad en el país y fortalecidas sus capacidades de Género y Comunicación. (PIEGH; 2010)

\section{2.- Principales desafíos de las mujeres en educación}

- Priorización de la implementación de las políticas y programas de igualdad y equidad en género y los recursos pertinentes en todas las instituciones del sistema educativo. 
- Establecer alianzas estratégicas con decisores, funcionarios y funcionarias del nivel intermedio y político del sector educación.

- Implementación de procesos de sensibilización a los decisores y funcionarios de los niveles operativos del sistema educativo.

- Promoción de la Política Nacional de la Mujer - II PIEGH en el ámbito educativo para la divulgación de los derechos de las mujeres y la igualdad de género a nivel nacional, regional y municipal.

- Promoción de alianzas y compromisos de las instituciones u organizaciones comunitarias para la igualdad y equidad de género y la protección de los derechos de las mujeres, las niñas y adolescentes. (PIEGH; 2010)

- Inclusión del enfoque de género, Derechos Humanos y prevención de violencia contra las mujeres y las niñas en los programas de sensibilización y capacitación docente para el desarrollo de conocimientos en el manejo de situaciones de vulneración de derechos.

\section{C.- MEDIDAS A TOMAR EN EL SISTEMA EDUCATIVO NACIONAL QUE PROPONEN LA EQUIDAD DE GÉNERO}

La categoría de equidad de género en la educación básica permite abordar con mayor propiedad la problemática de la desigualdad entre varones y mujeres, tanto en lo privado como en lo público; lo que se puede lograr con procesos de aprendizaje en todas las etapas de la vida que Ileven a los seres humanos a reconocer y respetar la diversidad, gozar de igualdad de valor, emprender acciones temporales para fomentar la equidad de género tales como:

- Eliminación de estereotipos sexistas

- Reconocimiento de las capacidades potenciales de cada persona

- Consideración de sus distintas condiciones de vida

- Valoración de manera equilibrada, las cualidades, intereses y necesidades.

- Procuración de un desarrollo pleno en los distintos ámbitos de la sociedad.

- Consensuar con todos los sectores la inclusión de la igualdad de género en todo el sistema educativo nacional. 


\section{CONCLUSIONES}

1.- Se necesitan mayores esfuerzos en la aplicación y eficacia de los instrumentos legales ya existentes para una mayor efectividad en su aplicación.

2.- Destacar la necesidad de mayores esfuerzos por implementar medidas y acciones afirmativas que permitan una mejor y mayor incorporación de las mujeres en la vida del país y conciliación de roles domésticos y productivos.

3.- Pensar siempre en obtener los mayores beneficios para las mujeres en los procesos de desarrollo, en particular las mujeres campesinos y de diferentes grupos étnicos, con el fin de darles mayores oportunidades para poder salir adelante.

4.- La desigualdad por razones de género constituye un obstáculo para el derecho a la educación y el desarrollo pleno de las mujeres, ya que conlleva a que se violen sus derechos como parte de la sociedad.

5.- A pesar del reconocimiento a la igualdad de derechos políticos de las mujeres desde 1955, todavía falta recorrer un largo trecho para vencer los obstáculos a la igualdad plena y hacer realidad aquello por lo que lucharon con todo ahínco los sufragistas hondureños.-

6.- Finalmente, a pesar del empoderamiento educativo que han logrado las mujeres, del desempeño exitoso de muchas mujeres en la empresa privada, la política, sectores sociales, arte y deportes, su presencia es todavía muy distante a la proporción de la que deberían gozar en los espacios representativos, órganos de gobierno y cuerpos colegiados y si consideramos que son casi el $51 \%$ de la población.-

\section{RECOMENDACIONES}

- Que el Sistema Educativo Nacional como agente reproductor de valores, normas, creencias y actitudes, no intervenga en la construcción de las desigualdades sociales y en particular las que atañen a relaciones entre las mujeres y los hombres, moldeando desde muy temprana edad la forma de concebir la realidad y las reglas de funcionamiento de la sociedad en la que vivimos. 
- Implementar Políticas que compatibilicen la vida familiar con el trabajo y el fortalecimiento de los valores de igualdad y respeto en materia de derechos humanos en distintas esferas culturales e institucionales, para que las mujeres se puedan empoderar de su rol como parte de la sociedad.

- Todos los gobiernos deben comprometerse a la elaboración de una política de equidad de género en el sector educación que oficialice la incorporación de la perspectiva de género como eje transversal, en la ley como en la práctica, porque de nada sirve predicar sin dar el ejemplo, por lo tanto las mujeres deben participar en todos los procesos de cambio que ayuden a mejorar su posición en la sociedad y así ser escuchada en los diferentes ámbitos de la misma. Por lo tanto debe prevalecer en las leyes la igualdad, la imparcialidad y el respeto hacia las mujeres.

- Invertir en la capacitación y sensibilización de los recursos humanos con que cuentan las instituciones del Estado, desde un enfoque de género, para que esto contribuya sin duda a garantizar el respeto de los Derechos Humanos Universales de todas las personas que viven en condiciones de desventaja social debido a la discriminación de las cuales son objetos.

\section{BIBLIOGRAFÍA}

- Acosta, Ana (2011, 25 de Noviembre) Informe 2011. Niñas de Honduras Sumergidas en Discriminación. Diario El Heraldo. P. 18

- Bernal, Cesar Augusto (Metodología de la Investigación. Editorial Pearson Education. D.F, México.

- "Conquistar la igualdad: la coeducación hoy" Dra. Marina Subirats, en la Revista Iberoamericana de Educación .Monográfico: "Género y Educación". Septiembre-Diciembre 1994. Citado por Op. Cit, Las Dignas.

- Cuarta Conferencia Mundial sobre la Mujer. (1995) Beijing.

- Editorial (2011, 8 de Marzo) Grandes Avances y Mucho por Recorrer. Diario El Heraldo. Pág. 39

- Fonseca, Regina (a.n.d) Memoria Foro: Mujeres en Espacios de Toma de Decisiones.2009 
- Gomáriz, Enrique. (1994) La Planificación con Perspectiva de Género. Colección Metodologías No. 1. CMF. San José, Costa Rica.

- II Plan de Equidad de Género de Honduras (PIEGH) 2010-2022 (2010) Tegucigalpa, Honduras.

- Informe Final. Foro Mundial sobre la Educación (Abril 2000) Dakar.

- Lagarde, Marcela. (1992) Identidad de Género. Editorial Cenzontle. Nicaragua.

- Montejo, Aldafacio. (1992) Cuando el Género suena cambios trae. Metodologías para análisis de género en el fenómeno legal. ILANUD. San José, Costa Rica.

- Redacción (2011, 11 de Mayo) Informe Rendimiento Académico, Niñas tienen mejor desempeño que los niños en todos los niveles. Diario El Heraldo. Pág. 4. 